\title{
DIA+: A data-independent acquisition method combines multiple precursor charges to improve peptide signal.
}

\author{
Eva Borràs ${ }^{1,2}$, Eduard Sabidón ${ }^{1,2, *}$ \\ ${ }^{1}$ Proteomics Unit, Center for Genomics Regulation, Barcelona Institute of Science and \\ Technology (BIST), Barcelona, Spain \\ ${ }^{2}$ Proteomics Unit, Universitat Pompeu Fabra, Barcelona, Spain \\ * Corresponding author \\ Eduard Sabidó \\ Proteomics Unit, Center for Genomics Regulation, Barcelona Institute of Science and \\ Technology (BIST), Universitat Pompeu Fabra, Barcelona, Spain \\ Dr. Aiguader 88 \\ 08003 Barcelona \\ eduard.sabido@crg.cat
}

To the Editor: Data-independent acquisition (DIA) methods that acquire fragment ions from virtually any peptide in a sample have expanded the benefits of low-throughput targeted proteomics to proteome-wide analyses. ${ }^{1}$ These methods rely on the use of several broadband isolation windows that select and fragment all peptides within each cycle and generate complex spectra containing fragment ions from multiple peptides. ${ }^{2}$ These data sets can then be analyzed in a targeted or untargeted manner to quantify virtually all peptides within a sample above the instrument detection limit. ${ }^{3,4}$ While these methods have increased the reproducibility of peptide quantification across multiple samples, their sensitivity is still limited, and the quantification of complete proteomes remains a challenge. ${ }^{5,6}$ Indeed, DIA methods currently need to balance sensitivity and selectivity with the covered $\mathrm{m} / \mathrm{z}$ range, and while narrow isolation windows result in higher selectivity, they limit the $\mathrm{m} / \mathrm{z}$ extension and thus, the number of quantified peptides and proteins in a single run.

Here we present DIA+, a novel DIA multiplexing scheme that combines signals from identical peptides with different charge states to improve sensitivity and selectivity while covering a wide $\mathrm{m} / \mathrm{z}$ range. DIA+ acquisition scheme relies on the isolation of three non-consecutive $\mathrm{m} / \mathrm{z}$ segments of $5 \mathrm{Da}, 7.5 \mathrm{Da}$ and $11.25 \mathrm{Da}$-based on the mass difference between charge +2 and +3 peptides-into a composite window of $23.75 \mathrm{Da}$, thus covering two sets of charge +3 and +2 precursor ion relationships in a single composite window. Forty of these composite windows are used to cover a wide range of 400-1350 m/z (Figure 1a). 
The performance of DIA+ was compared with two other reference methods in the field, the $20 \times 20$ method consisting in 20 windows of 20 Da covering the range of $500-900 \mathrm{~m} / \mathrm{z}$, and the $32 \times 25$ method consisting in 32 windows of 25 Da covering the range of $400-1200 \mathrm{~m} / \mathrm{z}$. Moreover, the DIA+ was also compared with a DIA+ control method (CTRL) in which each 23.75 Da composite window resulted from the combination of three consecutive $\mathrm{m} / \mathrm{z}$ segments (Supplementary Figure S1). To evaluate the DIA+ method, $1 \mu \mathrm{g}$ of tryptic peptides derived from digested HeLa cell extracts were injected in triplicates in an Orbitrap Fusion Lumos mass spectrometer with each of the tested methods using 120-min chromatographic gradients. All DIA methods were acquired with a first MS1 scan (Orbitrap detection at 60K) followed by different quadrupole isolation schemes, HCD fragmentation (28\% CE) and MS2 detection in the Orbitrap at 30K (Supplementary Methods). Data were analyzed using DIA Umpire v2.1 ${ }^{3,7}$ with the search engine Comet. Peptides and proteins were filtered at 1\% FDR and their quantification was based on the sum of the six most intense fragment ions and the six most intense peptides respectively.

In contrast to sequential or at-random non-consecutive multiplexing schemes ${ }^{8}$, DIA+ composite windows are designed to combine analytes at different charge states ( +2 and +3$)$. Therefore, in $\mathrm{DIA}+$ the signal obtained in each composite window combines information from each charge state, which i) reinforces the signal by summing signals from common fragments between charge states, and ii) complements the signal by including fragments that are unique to certain charge states. This increases the signal-to-noise ratio compared to other methods (Supplementary Figure S2), and results in a higher number of matched fragments per peptide as well as a better distribution of peptide scores and probabilities (Figure $\mathbf{1 b}$, and Supplementary Figure S3).

Moreover, since the combined windows in the DIA+ method are made of non-consecutive isolation segments, they probe different $\mathrm{m} / \mathrm{z}$ regions thus introducing a tiling effect and lowering the dependency between the number of precursors identified and the chromatographic gradient (Supplementary Figure S4). This peptide distribution contrasts with the other tested methods in which most of the time in each cycle is dedicated to the detection of ion packages coming from isolation windows with low peptide content. Additionally, the combination of isolation segments using the charge rationale reduces the number of analytes per composite window, thus increasing the specificity of the DIA+ method (Supplementary Figure S5). To discern tiling effects from the benefits of charge combination, a direct comparison was done among the isolation segments that are common between DIA+ and its control method (CTRL) (Supplementary Figure S6a). This comparison evidenced that DIA+ is outperforming the control method (CTRL) in each of these common segments, and that the gain in peptide quantification is not random, but enriched in either +2 or +3 precursors in agreement with the expected result when combining different charge states (Supplementary Figure S6b). 
Overall, DIA+ covers a wide $\mathrm{m} / \mathrm{z}$ range, and the improved signal-to-noise, the additional number of fragments, and the reduced window complexity aids automated algorithms-such as DIAUmpire-to recognize transition groups. Consequently, DIA+ increases the number of peptides identified and quantified when compared to other methods, with ca. $30 \%$ gain in respect to the CTRL method (Figure 1c). The data could be further queried using prior knowledge such as spectral libraries, a strategy that might increase the final number of identified and quantified peptides. However, to ensure a good match in relative intensities, spectral libraries should be generated with a similar acquisition scheme, otherwise spectra from peptides with charge +2 and +3 should be combined in-silico prior library usage.

In conclusion, DIA+ is a novel data independent acquisition method with improved sensitivity and selectivity, which results in a higher number of identified and quantified peptides and proteins in complex samples. DIA+ might benefit applications such as site-localization of posttranslational modifications and the identification of single-point mutations and polymorphisms.

The mass spectrometry proteomics data obtained from the DIA+ and the companion methods have been deposited at the ProteomeXChange Consortium via the PRIDE repository with identifier PXD010210. ${ }^{9}$

\section{Keywords}

DIA, Data-independent acquisition, charge, protein quantification

\section{Acknowledgements}

The CRG/UPF Proteomics Unit is member of ProteoRed PRB3 consortium which is supported by grant PT17/0019 of the PE I+D+i 2013-2016 from the Instituto de Salud Carlos III (ISCIII) and ERDF. We acknowledge support from the Spanish Ministry of Economy and Competitiveness, "Centro de Excelencia Severo Ochoa 2013-2017", SEV-2012-0208, and "Secretaria d'Universitats i Recerca del Departament d'Economia i Coneixement de la Generalitat de Catalunya" (2017SGR595).

\section{Supporting Information Available}

Supplementary Figures S1-S6

Supplementary Methods

- Mass spectrometry acquisition

- Acquisition Schema

- Data analysis parameters 


\section{Bibliography}

1. Bensimon, A., Heck, A. J. R. \& Aebersold, R. Mass spectrometry-based proteomics and network biology. Annu. Rev. Biochem. 81, 379-405 (2012).

2. Borràs, E. \& Sabidó, E. What is targeted proteomics? A concise revision of targeted acquisition and targeted data analysis in mass spectrometry. Proteomics 17, (2017).

3. Tsou, C. C., Avtonomov, D., Larsen, B., Tucholska, M., Choi, H., Gingras, A. C., \& Nesvizhskii, A. I. DIA-Umpire: comprehensive computational framework for data-independent acquisition proteomics. Nat. Methods 12, 258-264, 7 p following 264 (2015).

4. Gillet, L. C., Navarro, P., Tate, S., Röst, H., Selevsek, N., Reiter, L., Bonner, R., and Aebersold, R. Targeted data extraction of the MS/MS spectra generated by dataindependent acquisition: a new concept for consistent and accurate proteome analysis. Mol. Cell. Proteomics MCP 11, O111.016717 (2012).

5. Selevsek, N., Chang, C-Y., Gillet, L. C., Navarro, P., Bernhardt, O. M., Reiter, L., Cheng, LY., Vitek, O., and Aebersold, R. Reproducible and consistent quantification of the Saccharomyces cerevisiae proteome by SWATH-mass spectrometry. Mol. Cell. Proteomics MCP 14, 739-749 (2015).

6. Picotti, P., Bodenmiller, B., Mueller, L. N., Domon, B. \& Aebersold, R. Full dynamic range proteome analysis of S. cerevisiae by targeted proteomics. Cell 138, 795-806 (2009).

7. Tsou, C.-C., Tsai, C.-F., Teo, G. C., Chen, Y.-J. \& Nesvizhskii, A. I. Untargeted, spectral library-free analysis of data-independent acquisition proteomics data generated using Orbitrap mass spectrometers. Proteomics 16, 2257-2271 (2016).

8. Egertson, J. D., Kuehn, A., Merrihew, G. E., Bateman, N. W., MacLean, B. X., Ting, Y. S., Canterbury, J. D., Marsh, D. M., Kellmann, M., Zabrouskov, V., Wu, C. C., \& MacCoss, M. J. Multiplexed MS/MS for improved data-independent acquisition. Nat. Methods 10, 744-746 (2013).

9. Vizcaíno, J. A., Deutsch, E. W., Wang, R., Csordas, A., Reisinger, F., Ríos, D., José A Dianes, J. A., Sun, Z., Farrah, F., et al. ProteomeXchange provides globally coordinated proteomics data submission and dissemination. Nat. Biotechnol. 32, 223-226 (2014). 


\section{Figures}

Figure 1. a) DIA+ isolation scheme. Three non-consecutive $\mathrm{m} / \mathrm{z}$ segments of $5 \mathrm{Da}, 7.5 \mathrm{Da}$ and 11.25 Da are isolated into a composite window of 23.75 Da. Forty composite windows cover a range of $400-1350 \mathrm{~m} / \mathrm{z}$. Since these segments are based on the mass difference between charge +2 and +3 peptides, they cover two sets of charge +3 and +2 precursor ion relationships in a single composite window, i.e. the first (yellow) and second (red) segments cover one $+3 /+2$ relationship, whereas the second (red) and third (blue) cover another $+3 /+2$ relationship. The combination of signals from the same peptide species with different charge states show a cumulative signal in MS2. b) Density plots of the number of matched fragments per spectrum (Comet), and PeptideProphet probability of identified peptides separated by charge (z=1-4). c) Number of peptide ions and protein groups identified in DIA+, CTRL, 32X25 and 20X20 at 1\% FDR. 